\begin{tabular}{|c|c|}
\hline Editorial & \\
\hline $\begin{array}{l}\text { Neue Organisationsformen } \\
\text { erfordern neue Steuerungs- } \\
\text { werkzeuge }\end{array}$ & 81 \\
\hline Inhalt & 82 \\
\hline \multicolumn{2}{|l|}{ Notizen... } \\
\hline $\begin{array}{l}\text { aus dem Produktions- } \\
\text { technischen Zentrum Berlin }\end{array}$ & 84 \\
\hline $\begin{array}{l}\text { aus Branchen und } \\
\text { Unternehmen }\end{array}$ & 84 \\
\hline Kalender & 86 \\
\hline $\begin{array}{l}\text { Planen und Steuern der } \\
\text { Produktion }\end{array}$ & \\
\hline
\end{tabular}

M.Wassel, D. Küchler, K. Mertins,

R. Albrecht, S. Wilksch

Auftragssteuerung für schlanke Unternehmen

FLS - Forum für Anwender und Anbieter

\section{Moser}

Wettbewerbsvorteile durch moderne Fertigungsleittechnik

P. Scholtissek, J. Gläßer PPS auf dem Prüfstand - Simulationsgestützter Verfahrenstest

PPS-Komplettlösung für mittlere Betriebe

G. Daniëls

Merkmale von PPS-Systemen der neuen Generation

A. Kelemis

Wissensbasiertes Fertigungsleitsystem

\section{Feistenberger}

Ein PPS-System entsteht 104

NC-Steuerungsdaten simulieren und verifizieren

W. Hautsch

Digitale Leitstandstechnik in der Druckindustrie

PPS-spezifischer Fertigungsleitstand

Geschäftsabläufe in der Prozeßindustrie steuern

W. Winkelmann, B. Hufnagl BDE-Lösung für kleine und mittlere Fertigungsbetriebe

\section{H. Kruppke}

Leitstände für das Geschäftsprozeß-Management

Kostengünstiges PPS für kleine und mittlere Unternehmen 118

Warenwirtschaftssystem auf OS/2 verfügbar

\section{Kundenbezogenes Steuern der Produktion}

Den heutigen Anforderungen an die Fertigung ist nicht mit einem Mehr an übermächtiger Planung zu begegnen. Vielmehr giltes, Eigenverantwortlichkeit, prozeßnahes Entscheiden und unternehmerisches Denken in kleinen Teams auch in einem adäquaten Informationssystem abzubilden. Ein solches System basiert auf Hauptfunktionen wie Auftragseinsteuerung, Materialmanagement und Statistik, die in einem dezentralen Logistiksystem jeder Produktionseinheit zur Verfügung stehen.
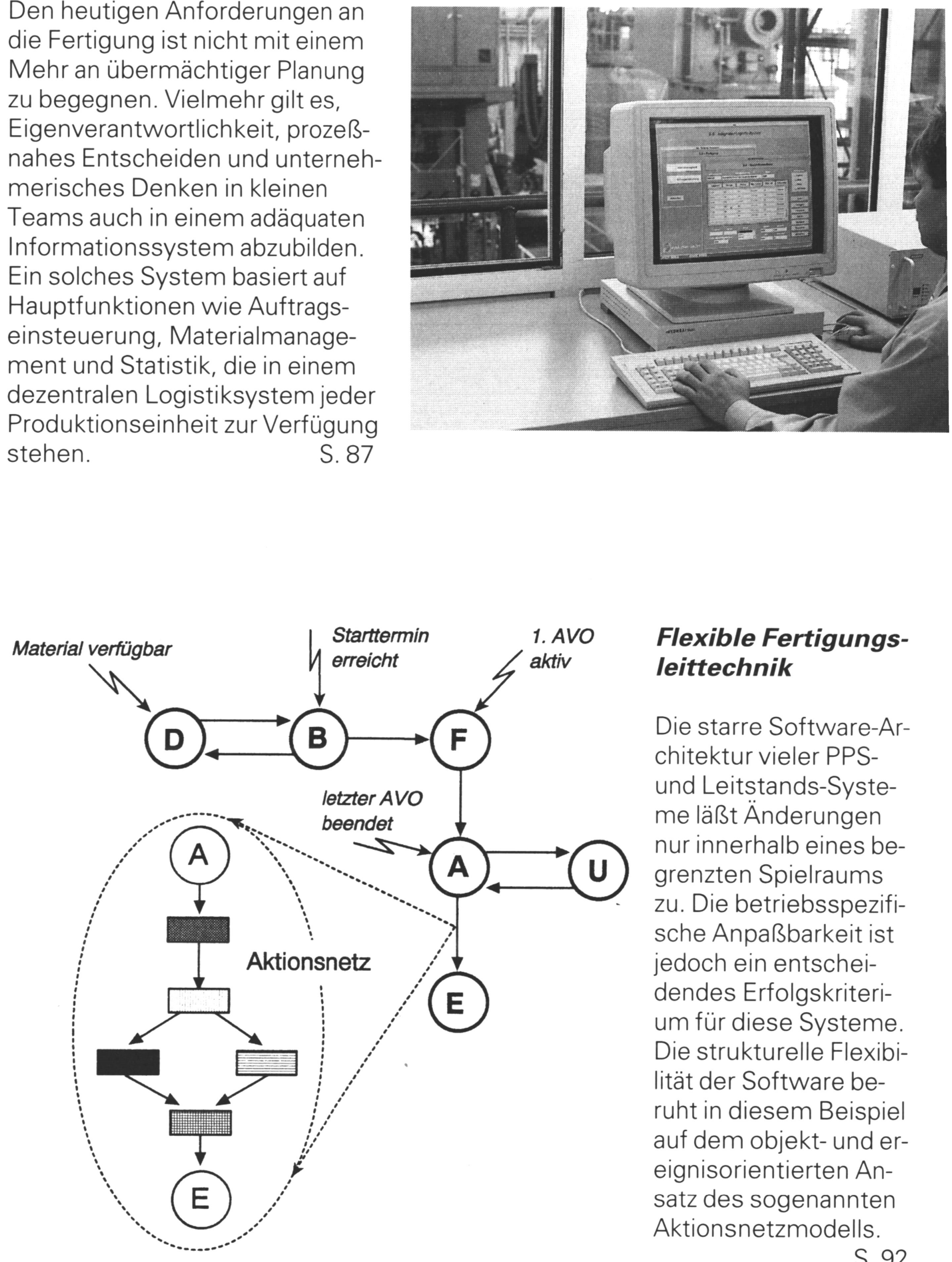

\section{Flexible Fertigungs- leittechnik}

Die starre Software-Architektur vieler PPSund Leitstands-Systeme läßt Änderungen nur innerhalb eines begrenzten Spielraums zu. Die betriebsspezifische Anpaßbarkeit ist jedoch ein entscheidendes Erfolgskriterium für diese Systeme. Die strukturelle Flexibilität der Software beruht in diesem Beispiel auf dem objekt- und ereignisorientierten Ansatz des sogenannten Aktionsnetzmodells.

\title{
PPS-Systeme simulieren
}

Mit Hilfe eines simulationsgestützten „PPSPrüfstands" ist eine anwendungsspezifische Überprüfung und Neukonfiguration von PPSVerfahren der betrachteten Produktionseinheit gleichsam im Probebetrieb möglich. Damit lassen sich ungenutzte logistische Potentiale erschließen.

S. 96

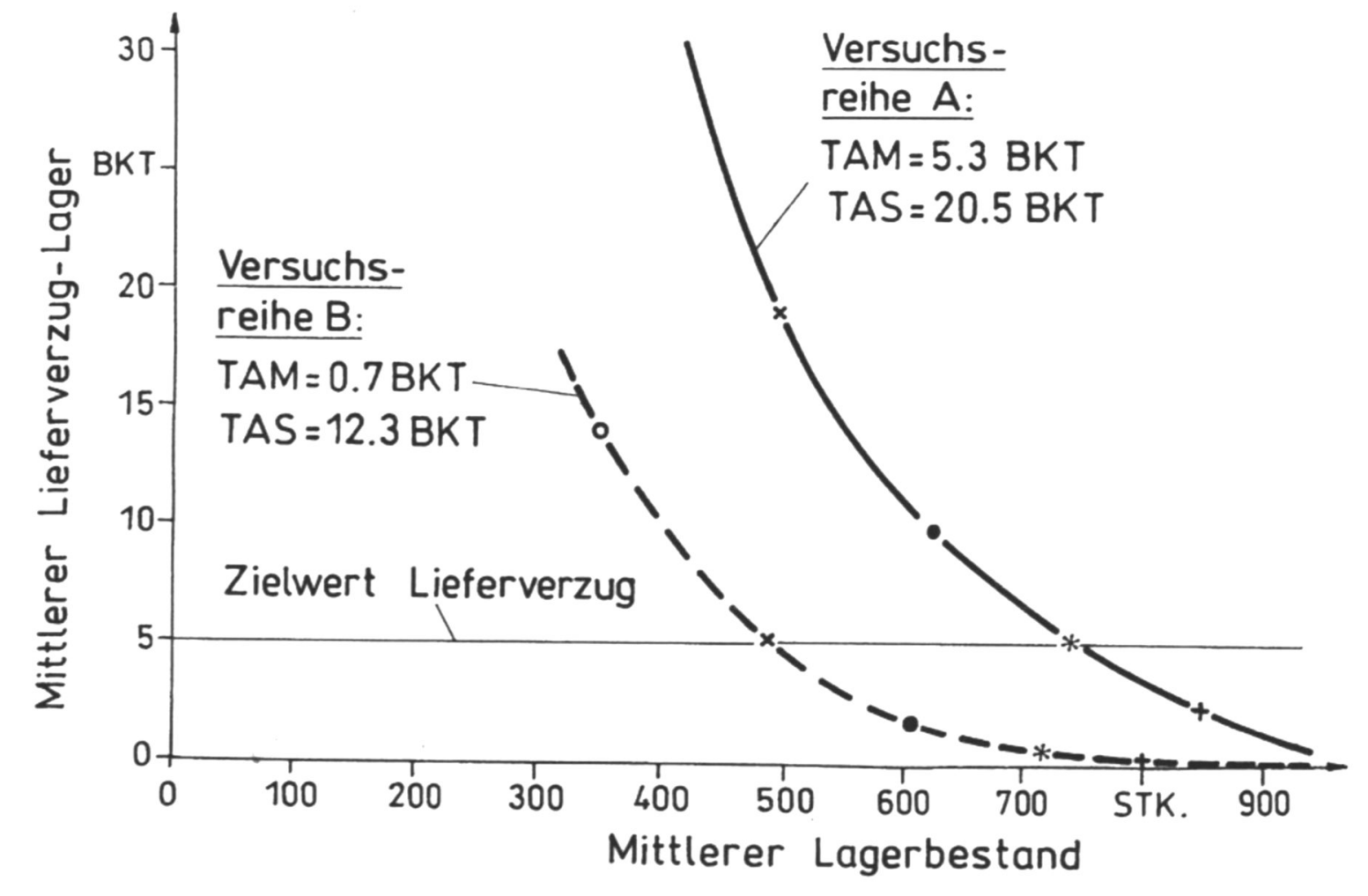




\section{Umsetzung strategischer Unternehmensziele}

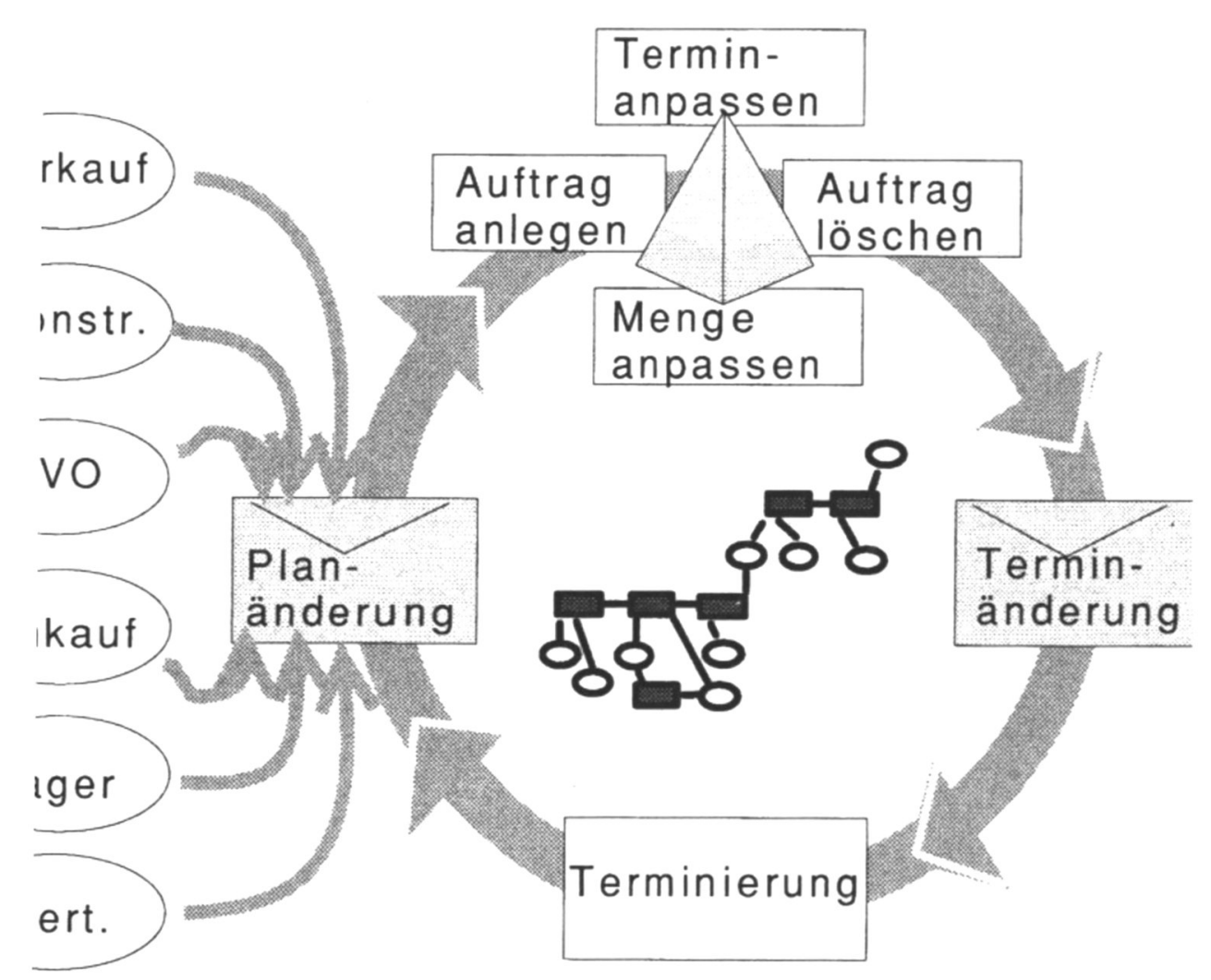

Das PPS-System ist keine absolutistische Steuerungsinstanz, sondern eine Einrichtung, die verschiedenen Bereichen Informationen und Dienstleistungen bietet. Dabei müssen sich je Produktgruppe, Auftragsart oder sogar Werk die betrieblichen Hauptziele - geringe Lagerbestände, Kapazitätsauslastung, Lieferbereitschaft und kurze Durchlaufzeiten - verschieden gewichten lassen, um bestimmte Unternehmensziele zu verwirklichen.

S. 99

\section{Von der Fertigungssteuerung zum Geschäftsprozeß-Management}

Nachdem Leitstandssysteme in den letzten Jahren zum Teil sehr komplex geworden sind, wird nun die Forderung nach einer für den Anwender transparenten Leitstandstechnik laut. Die Modellierung der Abläufe der Fertigungssteuerung in Form sogenannter erweiterter ereignisgesteuerter Prozeßketten bietet einen Ansatzpunkt dafür, über die eigentliche Fertigung hinaus das Management von Geschäftsprozessen im Unternehmen überhaupt zu unterstützen.
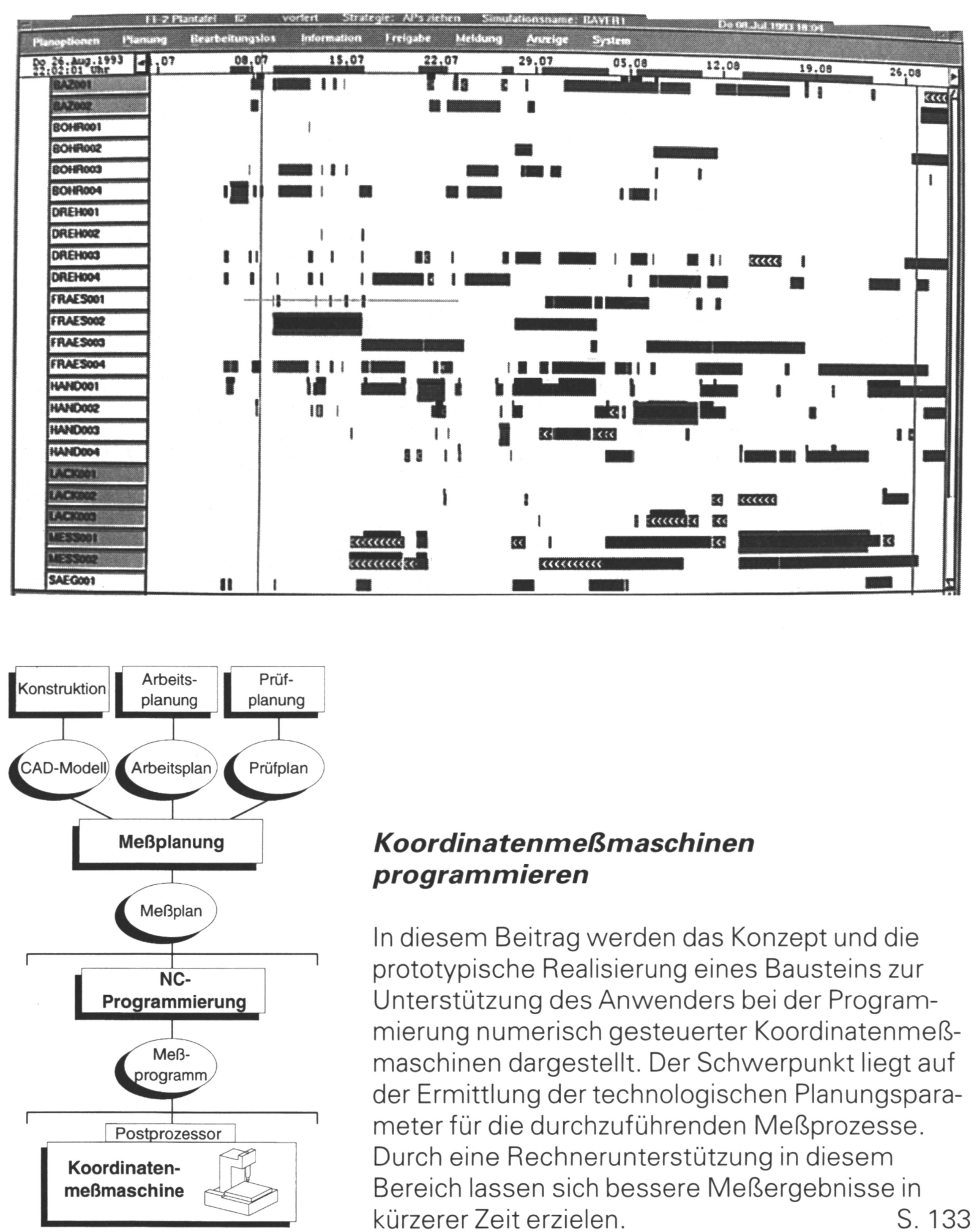

\section{Koordinatenmeßmaschinen programmieren}

In diesem Beitrag werden das Konzept und die prototypische Realisierung eines Bausteins zur Unterstützung des Anwenders bei der Programmierung numerisch gesteuerter Koordinatenmeßmaschinen dargestellt. Der Schwerpunkt liegt auf der Ermittlung der technologischen Planungsparameter für die durchzuführenden Meßprozesse. Durch eine Rechnerunterstützung in diesem Bereich lassen sich bessere Meßergebnisse in kürzerer Zeit erzielen.

A. Drexl, C. Jordan

Wissensbasierte Produktionsprozeßplanung

Geschäftsprozesse optimieren 121

H.-D. Haasis

PPS-Systeme und betrieblicher

Umweltschutz

Wieviel kostet Standardsoftware wirklich?

Fertigungsleitstand mit BDEIntegration

Rechnerunterstützte

Konstruktion und Planung

R. Volck, G. Wetzel

Neustrukturierung der Konstruktionsarbeit durch intelligente Produktmodelle

J. Gausemeier, T. Frank

A. Sabin

Integrierte Verarbeitung von

Wiederholteil-Daten

Datenbank mit interner Parallelverarbeitung

Dokumenten-Management für den Anlagenbau

F.-L. Krause, M. Ciesla

Technologische Planung von

Meßprozessen für Koordinatenmeßmaschinen

Fabrikanlagen und Materialfluß

H. Tempelmeier, J. Hesselbach, H. Kuhn

Analyse der Wirtschaftlichkeit störungsreduzierender Maßnahmen an einer Fließlinie

Buchbesprechungen 141

Report

CAD $\cdot$ CAM $\cdot$ CIM

Fabrikanlagen und Materialfluß

\begin{tabular}{lr} 
Impressum & 144 \\
\hline Mit Sonderteil: & \\
CAD C CAM $\cdot$ CIM & CA 1-CA 48
\end{tabular}

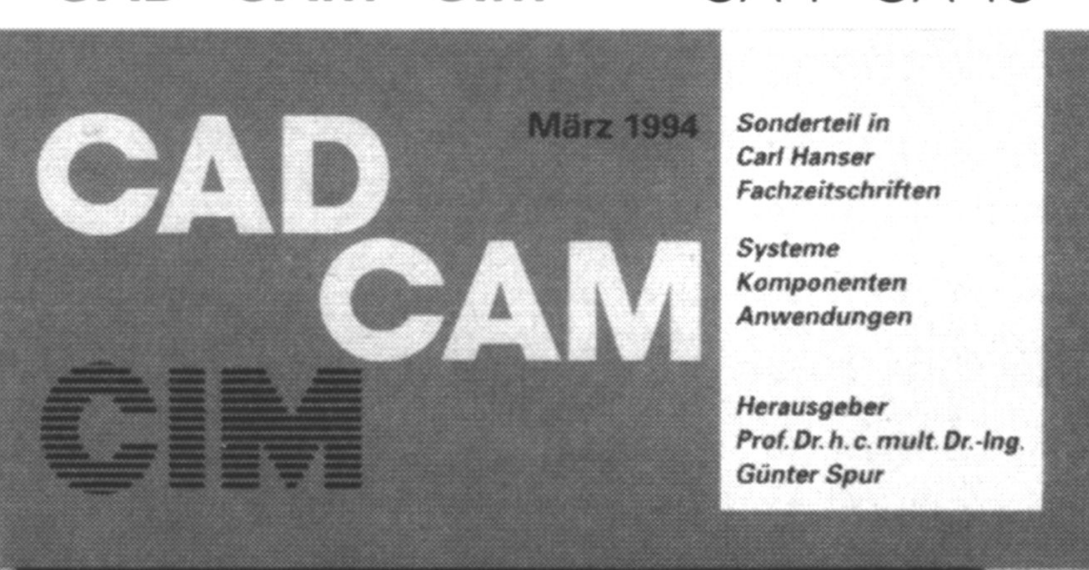

